\title{
Erfahrungsbericht der Autorin des Buches «Du hättest leben können»* Über Medizinfehler sprechen und daraus lernen
}

Stefanie Bachstein

* «Du hättest leben können», das Buch von Stefanie Bachstein mit einem Vorwort von Prof. Dr. med. Thomas H. Loew, Universitätsklinikum Regensburg, wurde unte einem Pseudonym veröffentlicht. In dem authentischen Erfahrungsbericht wie auch im folgenden Artikel wurden Namen, Daten, Ortsangaben und manchmal auch das Geschlecht verändert, um die zu schützen, deren Leben es betrifft.

Im Rahmen des Publizistikpreises 2004 der Stiftung Gesundheit in Hamburg erhielt das Buch eine besondere Erwähnung, weil Frau Bachstein auf die Traumatisierung beider Seiten aufmerksam macht.

horst@stefanie-bachstein.de

www.stefanie-bachstein.de

\section{Frühjahr 1996, Tod unserer kleinen Tochter durch einen Medizinfehler}

Unsere siebenjährige Jule hatte auf dem Schulweg einen Verkehrsunfall. Dabei wurde sie vom Aussenspiegel eines fahrenden PKW im Gesicht getroffen. Rettungswagen und Notarztwagen waren in kürzester Zeit am Unfallort. Eine Fehleinschätzung der Lage und eine unerkannte Fehlintubation der erstversorgenden Notärztin haben laut gerichtsmedizinischem Gutachten, das von der Staatsanwaltschaft in Auftrag gegeben wurde, den Tod unseres Kindes verursacht. Die Notärztin hatte unter der Verdachtsdiagnose «schweres Schädel-Hirn-Trauma» eine Intubation vorgenommen. Unser Kind war durch Medikamente relaxiert und die Spontanatmung somit ausgeschaltet. Ein Rettungshubschrauber war angefordert.

Als der herbeigerufene Hubschraubernotarzt, ein Anästhesist, eintraf, sah er sofort, dass Jule fehlintubiert war. Trotz aller Anstrengung konnte er ihr Leben nicht retten. Jule starb im Hubschrauber auf dem Flug ins nächste grössere Klinikum. Der Hubschraubernotarzt und die Klinik vertuschten den folgenschweren Fehler ihrer Kollegin nicht. Dafür sind wir bis heute dankbar.

Der diensthabende Klinikarzt teilte uns Eltern mit, dass Jule gestorben sei, und informierte uns wahrheitsgemäss, dass eine Fehlintubation vorläge. Deshalb sei die Staatsanwaltschaft eingeschaltet und eine gerichtsmedizinische Obduktion angeordnet, gegen die wir Eltern nichts unternehmen könnten. Um das rechtsmedizinische Gutachten zu erhalten, müssten wir uns einen Anwalt nehmen. Ich war so im Schock, dass ich nur verstand «Jule ist tot»was eine Fehlintubation ist, wusste ich damals nicht.

Als mir befreundete Ärzte am Nachmittag erklärten, was das bedeutet, geriet ich in Panik. So durfte mein Kind nicht gestorben sein, das würde ich nicht verkraften. Das durfte einfach nicht wahr sein. Ich zog mich innerlich auf die Position zurück: «Jule wäre sowieso gestorben an ihren schweren Verletzungen.»

Ich kann mir vorstellen, dass diese Schutzhaltung auch bei der jungen Notärztin, die damals als Assistenzärztin in einer Klinik arbeitete, in ähnlicher Weise vorhanden war. Zum Tod unseres Kindes erhielten wir von der Notärztin und ihrem Team oder ihrem Vorgesetzten kein Zeichen des Bedauerns. Das wäre wichtig gewesen. Statt dessen Schweigen.

\section{Ein halbes Jahr später, Herbst 1996, das gerichtsmedizinische Gutachten}

Nach Jules Tod habe ich psychologische Hilfe in Anspruch genommen und ein halbes Jahr intensiv um mein Kind getrauert. Als wir mit unseren beiden Söhnen, damals 10 und 12 Jahre alt, aus unserem ersten Sommerurlaub ohne Jule zurückkamen, lag das gerichtsmedizinische Gutachten in unserem Briefkasten. Schwarz auf weiss lasen wir, dass Jule eindeutig durch die unerkannte Fehlintubation gestorben war. Das Gutachten ging von 7 bis 10 Minuten Fehlintubation aus. Gegen die Ärztin wurde weiter wegen fahrlässiger Tötung ermittelt. Das Rettungsteam hatte bei der Vernehmung durch die Kriminalpolizei zu Protokoll gegeben, dass niemand die Fehlintubation in die Speiseröhre bemerkt habe.

Obwohl Jule schon ein halbes Jahr tot war, musste ich mich nun der Tatsache stellen, dass unser Kind durch einen vermeidbaren Behandlungsfehler gestorben war.

Ich suchte das Gespräch mit der Ärztin. Wir verabredeten uns in ihrem Büro. Bei diesem ersten Treffen versuchte Frau Dr. Fiedler mir klarzumachen, dass sie sich in dem gerichtsmedizinischen Gutachten ungerecht beurteilt fühle.

Ich spürte die Wut in mir hochsteigen, doch ich wollte nicht mit ihr streiten, sondern sie als Mensch kennenlernen und lenkte das Gespräch in eine andere Richtung. So kam es, dass sie während dieser Begegnung zwei Sätze sagte, die mich tief berührten. Auf die Frage «Wie kam es, dass Sie Medizin studierten?» antwortete sie: «Ich wollte schon immer Ärztin werden, solange ich denken kann. Das war mein Traumberuf. Und dadurch ist jetzt soviel Elend entstanden.» Und weiter fuhr sie fort: «Ich bin schon zweimal durch Ihre Strasse gefahren, aber ich habe mich zu sehr geschämt, um bei Ihnen zu klingeln.» Auf meine Frage, ob sie jemanden habe, der sie in ihrer jetzigen Lage unterstütze, antwortete sie: «Nicht wirklich, meine Kollegen sagen schon 
mal 〈Kopf hoch〉 oder so etwas in der Art. Mein Chef Dr. Adams meint, ich solle ganz normal weiterarbeiten, auch als Notärztin, und so tun, als sei nichts geschehen.» Dann schaute sie mir in die Augen und sagte: «Es ist verrückt, aber manchmal habe ich, wenn ich mit dem Auto an einer Kreuzung warten muss, eine wahnsinnige Angst, Sie könnten neben mir halten. Ich bekomme dann Herzklopfen und Schweissausbrüche. Ähnlich ist es, wenn ich durch die Strasse fahren muss, in der der Unfall passierte.»

Frau Dr. Fiedler war nicht die abgebrühte Medizinerin, die das alles nichts anging. Ich reichte ihr zum Abschied in einer sehr bewussten Geste die Hand. Dieses Gespräch bewog mich, die Notärztin zu schützen, wo ich es vermochte, z. B. indem ich die Medien nicht informierte und ihren Namen nicht preisgab.

\section{Ein Jahr später, Frühjahr 1997, Verurteilung der Ärztin wegen fahrlässiger Tötung}

Der erste Todestag unseres Kindes im Frühjahr 1997 rückte näher. Ein schwerer Tag für uns alle. Ich musste immer wieder an Frau Dr. Fiedler denken, denn ich hatte gehört, dass sie arbeitsunfähig sei. Ich rief sie an. Gut aufgelegt erklärte mir die Ärztin, dass sie das Ganze jetzt überwunden habe und beim besten Willen nicht wisse, wo sie bei meiner Tochter etwas falsch gemacht habe. Ich war erschüttert - wie konnte sie nur so etwas sagen? Weil ich nicht in meinen Ohnmachtsgefühlen ertrinken wollte, entschloss ich mich, der Ärztin einen Brief zu schreiben, um sie zur Besinnung zu bringen. Ich forderte sie auf, ihre Approbation freiwillig zurückzugeben, da sie in meinen Augen ein Sicherheitsrisiko für zukünftige Patienten sei, wenn sie den Behandlungsfehler weiterhin verdränge.

Kurz danach wurde Frau Dr. Fiedler strafrechtlich wegen fahrlässiger Tötung $\mathrm{zu}$ einer Geldstrafe verurteilt. Nach dieser rechtskräftigen Verurteilung erhielten wir einen Brief ihres Vorgesetzten, des Chefarztes der Klinik, zu dem Dr. Fiedler ratsuchend mit meinem aufrüttelnden Brief gegangen war. Herr Dr. Adams schrieb, dass er sich persönlich kundig gemacht habe und zu dem Ergebnis gekommen sei, dass seiner Assistenzärztin kein Fehlverhalten vorzuwerfen sei. Es habe sich um einen schicksalhaften Unfallverlauf bei unserer Tochter gehandelt. Unsere Ohnmacht bezüglich eines Systems, in dem es diesen Fehler nicht geben «durfte», steigerte sich ins Unermessliche. Ich kam innerlich nicht mehr zur Ruhe. Damals begann ich, zu schreiben und meine Gefühle in Worte zu fassen: «Meine ohnmächtige Wut entsteht genau an der Stelle, wo niemand zu dieser Tragik stehen kann. Was wir zusätzlich zu unserer Trauer erleiden, geht über meine Kräfte. Ich fühle mich hintergangen, nicht ernst genommen, für dumm verkauft ...» Das zweite Trauma.

Ich hatte nicht allein den Tod meines Kindes zu verschmerzen, was immer eine traumatische Erfahrung ist, sondern ich war gezwungen, auch das Leugnen und Schönreden des Medizinfehlers zu verkraften. Mit der Ärztin empfand ich nach wie vor Mit-Schmerz, denn offensichtlich war ihr ein Schweigegebot auferlegt worden. Meiner Meinung nach wurde sie von ihren Vorgesetzten, Kollegen und ihrer Arzthaftpflichtversicherung allein gelassen, was die menschliche Seite und die psychische Aufarbeitung des tragischen Geschehens betraf. Auf unserem Rücken und auf dem der Ärztin sollte ausgebadet werden, was grundsätzlich nicht angeschaut werden durfte: ein ärztlicher Behandlungsfehler! Es schien um das Image eines Berufsstandes, einer Klinik und um Geld zu gehen, aber nicht um die Ärztin oder um uns als vom Schicksal schwer getroffene Menschen, geschweige denn um eine wahrhaftige Fehleranalyse, um daraus zu lernen.

\section{Zwei Jahre später - Frühjahr 1998}

Kurz vor Jules zweitem Todestag lag ein Kuvert mit einer mir unbekannten Handschrift in unserem Briefkasten. Ein persönlicher, zu Herzen gehender Brief von Frau Dr. Fiedler, in dem sie ihr Bedauern aussprach, auch was das Leugnen des Behandlungsfehlers bei unserem Telefonat ein Jahr zuvor betraf.

Ich konnte darauf nicht antworten, erst viel später schrieb ich: «Ihr Brief hat mir gezeigt, wie traurig Sie im Tiefsten Ihres Herzens sind. Ehrlich, ich habe nie etwas anderes vermutet. Es wäre gut gewesen, wenn wir das von Anfang an aus Ihrem Mund gehört hätten.»

Immer deutlicher nahm ich das Gesamtsystem wahr und wehrte mich. Der Klinikchef, Herr Dr. Adams, wurde durch unseren Anwalt veranlasst, eine Unterlassungsverpflichtungserklärung zu unterschreiben, denn wir wollten nicht unwidersprochen hinnehmen, dass er den Tod unseres Kindes als «schicksalhaften Verlauf» bezeichnete.

\section{Drei Jahre später, Ende 1999, zivilrechtliche Auseinandersetzung mit der Arzthaftpflichtversicherung der Notärztin}

Die Versicherung spielte auf Zeit und stellte den Behandlungsfehler permanent in Abrede. Das ist deshalb möglich, weil Strafrecht und Zivilrecht 
in Deutschland getrennt sind. Gefühlsmässig war das schwer zu verkraften. Unser persönliches Leid wurde heruntergespielt und bagatellisiert. Was die Arzthaftpflichtversicherung mit uns, aber insbesondere mit ihrer eigenen Versicherungsnehmerin, der Ärztin, gemacht hat, ist meines Erachtens haarsträubend, denn die Medizinerin zahlt monatlich deswegen ihren Versicherungsbeitrag. Die junge Medizinerin konnte sich nicht wehren, weil sie von ihrer Versicherung abhängig war, ich aber schon.

Mein Mann und ich wollten einen angemessenen Vergleich, ohne einen jahrelangen Zivilprozess gegen die Ärztin führen zu müssen. Doch die Versicherung forderte einen solchen geradezu heraus, und wir gerieten unter Zeitdruck. Die Verjährungsfrist von drei Jahren drohte abzulaufen. Kurz vor diesem Zeitpunkt entschlossen sich mein Mann und unser Anwalt zum Handeln. Sie wollten die Klage gegen die Ärztin einreichen. Ich spürte, dass das nicht gut wäre für unsere Familie. Über acht bis zehn Jahre würden wir durch alle Instanzen gezogen, und in dieser Zeit könnten weder wir noch unsere beiden Söhne das Geschehene verarbeiten und zur Ruhe kommen. Ebenso die junge Assistenzärztin. Das wollte ich verhindern. Um doch noch zu einer Lösung zu kommen, rief ich in der Hauptgeschäftsstelle der Versicherung in Deutschland an und wollte den Vorstandsvorsitzenden sprechen. «Ich sehe keine andere Möglichkeit mehr, als in grossem Stil an die Öffentlichkeit zu gehen», sagte ich der Chefsekretärin, die mich bat, meine Beschwerde schriftlich einzureichen, da ihr Chef leider nicht zu sprechen sei. Eine halbe Stunde später, noch während ich den Brief schrieb, erhielt ich einen Anruf unseres Anwalts. Der Sachbearbeiter der Versicherung habe ihn gerade verständigt, dass man nun doch zu einem Vergleich bereit sei. Eine mehr als erstaunliche Wendung - oder eben gerade nicht?

Als ich die Notärztin informierte, dass es gelungen sei, einen Zivilprozess zu verhindern, erzählte sie, dass sie ihrer Versicherung privates Geld angeboten habe, damit dieser Vergleich endlich zustande käme. Der Sachbearbeiter habe geantwortet: «Behalten Sie Ihr Geld, und lassen Sie diese Leute ruhig klagen.» Wenige Tage zuvor sei sie nun aufgefordert worden, die nicht geringe Summe dennoch zu überweisen, damit man den
Vergleich schliessen könne. Das habe sie gemacht.

Resigniert liess die Ärztin durchblicken, sie fühle sich von ihrer eigenen Versicherung mehr als im Stich gelassen.

Niemand, aber auch niemand konnte oder wollte mit diesem tragischen Geschehen angemessen umgehen. Das Einfachste und Normalste, nämlich zu sagen: «Hier ist etwas Schreckliches passiert, das niemals hätte geschehen dürfen, wir sind mit Ihnen fassungslos und erschüttert - es tut uns leid (oder ähnliches)», konnte keiner sagen. Das hat unser Leid zusätzlich zum Schmerz um unser Kind übermässig verstärkt. Traumatologen sprechen von einer Sekundär- oder Mehrfachtraumatisierung, die schwerer zu bewältigen war als die Trauer um unser Kind. Das gilt meines Erachtens auch für die Ärztin. Diese «Politik des Schweigens» könnte auch der jungen Ärztin den psychischen Verarbeitungsprozess sehr erschwert bis unmöglich gemacht haben.

Im Abschlussschreiben der renommierten Arzthaftpflichtversicherung lautete der Schlusssatz: «Vereinbarungsgemäss gehen wir davon aus, dass sich Familie Bachstein einer weiteren Äusserung in der Öffentlichkeit enthält [...].»

Das war ziemlich dicke Post: Wir hatten nichts vereinbart.

\section{Was für uns hilfreich gewesen wäre}

\section{Wahrhaftigkeit und Offenheit}

Wenn die Ärztin zu uns gekommen wäre und zu ihrem Fehler hätte stehen können und dürfen.

\section{Mit-Schmerz und Mit-Trauer}

Wenn die eigene Betroffenheit und Trauer für uns spürbar geworden wäre, auch von Vorgesetzten oder Kollegen.

\section{Die Zusage an uns Eltern, aus dem Fehler zu lernen}

Zum Beispiel durch eine genaue Fehleranalyse, denn aus einem Fehler, der nicht angeschaut werden darf, kann niemand lernen. Ich aber wünsche mir, dass aus Jules Tod Gutes wächst. 